\title{
INFLUENCE OF HEAT TREATMENT ON CORROSION PROPERTIES OF NON-ALLOY STEEL IN ACIDIC ENVIRONMENT
}

\author{
Sylwia Polasz \\ Gdynia Maritime University, Faculty of Marine Engineering \\ Department of Marine Maintenance \\ Morska Street 81-87, 81-225 Gdynia, Poland \\ tel.: +48585586564, fax.: +48585586432 \\ e-mail: s.polasz@wm.umg.edu.pl
}

\begin{abstract}
The article presents the influence of heat treatment on corrosion properties of non-alloy steel. This steel is used in machine elements. Heat treatment has a major impact on corrosion resistance of steel materials. Laboratory methods for measuring the corrosion rate consist of Electrochemical Impedance Spectroscopy and polarization curves. Instead of these types, there are also industrial methods of velocity corrosion measurements like researches in natural conditions and coupons corrosimetry. These days the most popular techniques of corrosion measurements are electrochemical measurements. In order to conduct impact assessments influent of heat treatment to corrosion properties there is need to proceed few steps like prepare samples, carry out heat treatment, hardness measurements, metallographical examination, prepare samples for corrosion and proceed corrosion researches by potentiodynamic method. An important element during researches is corrosion allowance, which has an impact influent on corrosion properties. This is the most popular and efficient method of corrosion protection. These days for corrosion protection are use paints or zinc coatings, cathode protection or stainless.
\end{abstract}

Keywords: corrosion process, non-alloy steel, corrosion preventive coatings, heat treatment, corrosion allowance

\section{Introduction}

These days, corrosion causes economic losses. Present materials engineering has introduced modern technological solutions for materials and plastics constructions. It is strived to ensure possible the longest durability of materials. To achieve this, it is necessary to make them resistant to corrosive environments.

Corrosion is the process of destroying metals or alloys in the solid state, under the influence of a chemical or electrochemical agent. It begins on the surface of metals and gradually goes deeper. When this process begins, it should be stopped as soon as possible. Otherwise, the metal element may be completely destroyed.

Corrosion resistance of metals and alloys can be increased by changing their composition by introduction of alloy additives and structure by using heat treatment or by eliminating internal tensions. Depending on the factors, affecting the corrosion process can be identified: chemical corrosion and electrochemical corrosion.

Heat treatment is a kind of technological process where the mechanical and physicochemical properties of metals and alloys are changed by causing modifications in their structure. The temperature, time, and environment have the main impact on this structure.

The most modern methods of measuring the corrosion rate are electrochemical impedance spectroscopy and electrochemical measurement of polarization curves.

\section{The experimental research methodology}

In order to assess the influence of heat treatment of corrosion properties of non - alloy steel 45 , tests were carried out. The scope included: 
I. Preparation of samples.

II. Performing heat treatment.

III. Hardness measurements.

IV. Preparation of samples for metallographic defects.

V. Metallographic research.

VI. Preparation of samples for corrosion tests.

VII. Performing corrosion tests by potentiodynamic method.

Samples with a diameter of $30 \mathrm{~mm}$ and a thickness of $10 \mathrm{~mm}$ were cut by a frame saw from round, unheated hot-rolled hotplate with an average carbon content of $0.45 \%$ and a maximum manganese content of $0.80 \%$.

The samples were subjected to the following heat treatment operations:

a. complete annealing at $830^{\circ} \mathrm{C}$,

b. incomplete annealing at $750^{\circ} \mathrm{C}$,

c. normalizing annealing at $830^{\circ} \mathrm{C}$,

d. martensitic hardening at $830^{\circ} \mathrm{C}$,

e. martensitic hardening and tempering at $250^{\circ} \mathrm{C}$,

f. martensitic hardening and tempering at $400^{\circ} \mathrm{C}$,

g. martensitic hardening and tempering at $650^{\circ} \mathrm{C}$.

The austenitizing temperature was selected on the basis of heat treatment and chemical composition of the processed material (phase equilibrium diagram $\mathrm{Fe}-\mathrm{Fe}_{3} \mathrm{C}$ ). The tempering parameters were selected based on literature data.

The hardness measurements were carried out on a Qness Q250M universal hardness device using a Vickers pyramid - shaped diamond indenter with an angle of 136 degrees at a load of $50 \mathrm{~kg}$ with $4 \times$ magnification. The measurement results were read from the device screen.

The corrosion research was tested on the Atlas Sollich 0531 Electrochemical Unit \& Impedance Analyser potentiostat. The measurements were carried out in an acid environment of $0.01 \mathrm{H}_{2} \mathrm{SO}_{4}$ at ambient temperature with calomel electrodes. The potential stabilization time was 30 minutes and the polarization range was $E_{k o r} \pm 150 \mathrm{mV}$. It allowed obtaining current information of the corrosion process. To determine the analysed parameters of the corrosive process (corrosive current density, corrosion potential) was used the Elfit program which performs calculations of polarization current density (1). Obtained results of measurements were obtained in the form of dependence plots $E=\log \left(I_{k o r}\right)$. The program performs calculations based on the equation:

$$
I=I_{k o r}\left[\left(1-\frac{I_{a}}{I_{d a}}\right) \exp \left(\frac{2.3\left(E-E_{k o r}\right)}{b_{a}}\right)-\left(1-\left|\frac{I_{k}}{I_{d k}}\right|\right) \exp \left(\frac{-2.3\left(E-E_{k o r}\right)}{b_{k}}\right),\right.
$$

where:

I - polarization current density $\left[\mu \mathrm{A} / \mathrm{cm}^{2}\right]$,

$I_{\text {kor }}$ - corrosive current density $\left[\mu \mathrm{A} / \mathrm{cm}^{2}\right]$,

$I_{a}$ - density of the partial current of the anode reaction $\left[\mu \mathrm{A} / \mathrm{cm}^{2}\right]$,

$I_{d a}$ - density of the anodic boundary current (diffusion) $\left[\mu \mathrm{A} / \mathrm{cm}^{2}\right]$,

$E-$ polarization potential $[\mathrm{mV}]$,

$E_{k o r}$ - corrosion potential [mV],

$b_{a} \quad$ - anode Tafel coefficient $[\mathrm{mV}]$,

$I_{d k}-$ density of the anodic boundary current (diffusion) $\left[\mu \mathrm{A} / \mathrm{cm}^{2}\right]$.

\section{The results of experimental research}

The raw hardness of the C45 sample is $170 \mathrm{HV}$. Obtained results of measurements can be found in Tab. 1. Fig. 1 presents a graph of the correlation between heat treatment and the obtained hardness. 
Tab. 1. Hardness results of samples (HV 50)

\begin{tabular}{|c|c|c|c|}
\hline No. & Hardness [HV] & No. & Hardness [HV] \\
\hline \multicolumn{2}{|c|}{ Incomplete annealing } & & Martensitic hardening and tempering at $250^{\circ} \mathrm{C}$ \\
\hline 1. & 168 & 16. & 555 \\
\hline 2. & 174 & 17. & 596 \\
\hline 3. & 175 & 18. & 592 \\
\hline 4. & 176 & 19. & 587 \\
\hline 5. & 179 & 20. & 588 \\
\hline \multicolumn{2}{|c|}{ Normalizing annealing } & & Martensitic hardening and tempering at $400^{\circ} \mathrm{C}$ \\
\hline 6. & 178 & 21. & 455 \\
\hline 7. & 188 & 22. & 449 \\
\hline 8. & 186 & 23. & 455 \\
\hline 9. & 185 & 24. & 456 \\
\hline 10. & 184 & 25. & 458 \\
\hline \multicolumn{2}{|c|}{ Complete annealing } & & Martensitic hardening and tempering at $650^{\circ} \mathrm{C}$ \\
\hline 11. & 156 & 26. & 244 \\
\hline 12. & 165 & 27. & 243 \\
\hline 13. & 167 & 28. & 242 \\
\hline 14. & 170 & 29. & 213 \\
\hline 15. & 171 & 30. & 244 \\
\hline \multicolumn{4}{|c|}{ Martensitic hardening } \\
\hline 31. & & & 720 \\
\hline 32. & & & 739 \\
\hline 33. & & & 788 \\
\hline 34. & & & 684 \\
\hline 35. & & & 684 \\
\hline
\end{tabular}

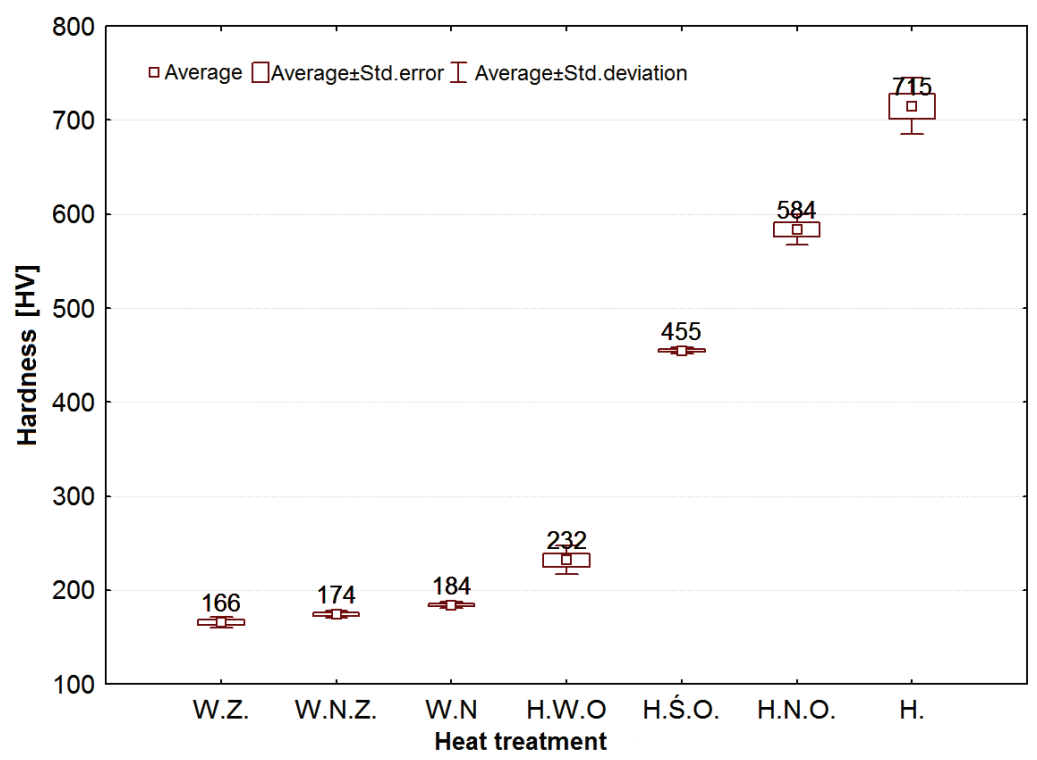

Fig. 1. Hardness dependence of C45 steel on heat treatment; W.Z. - complete annealing, W.N.Z. - incomplete annealing, W.N. - normalizing annealing, H.W.O. - high tempered hardening H.Ś.O. - medium tempered hardening, H.N.O. - low tempered hardening, H. - martensitic hardening

The structures of the performed tests are shown in Fig. 2 and 3. The structure in the sample after incomplete annealing is characterized by bright ferrite grains and dark perlite. The ferrite grains are larger than the average size of perlite grains. In the sample after complete annealing at 
$850^{\circ} \mathrm{C}$, ferrite and perlite are visible with a coarse structure. On the sample after normalizing, grains of ferrite and perlite are also visible but the grain structure is finer. In the sample after martensitic hardening, coniferous martensite grains and a small amount of residual austenite are visible. The sample after martensitic hardening and tempering at $250^{\circ} \mathrm{C}$ shows the coniferous structure of martensite with precipitation of cementite. On the surface of the sample after martensitic hardening and tempering at $400^{\circ} \mathrm{C}$ can be notice the coniferous structure of martensite and the fine pearlite. The sample after martensitic hardening and tempering at $650^{\circ} \mathrm{C}$ shows the coniferous martensite structure and the sorbitol. The precipitation of cementite is also visible.

a)

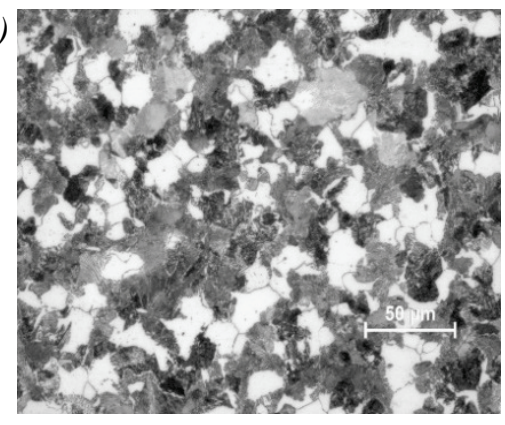

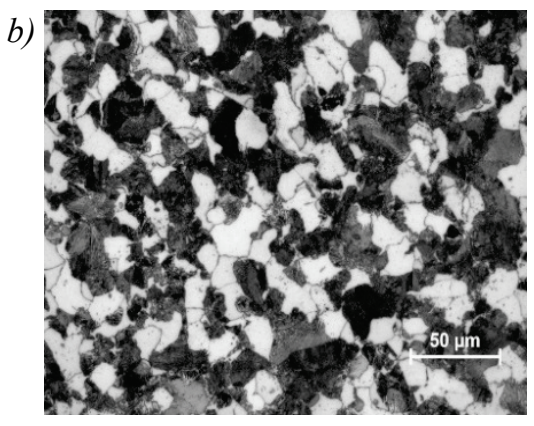

Fig. 2. Microstructure of C45 steel subjected to annealing treatment: a) incomplete, b) complete, c) normalizing

a)

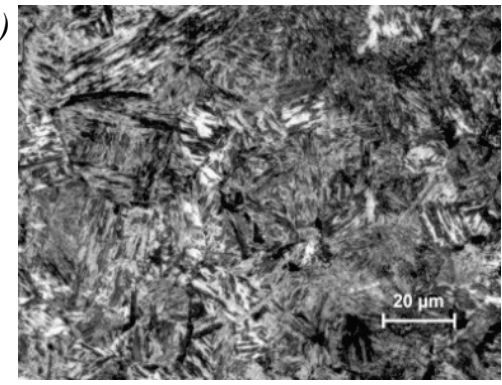

c)

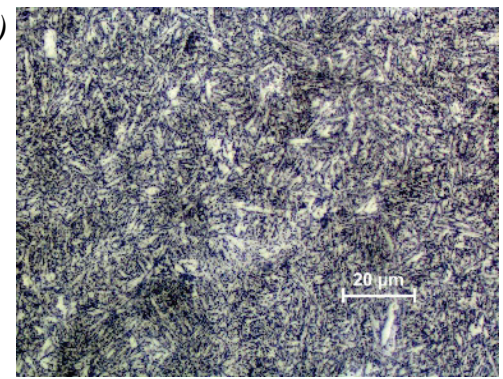

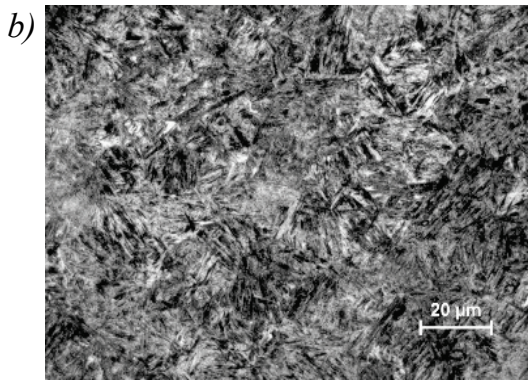

d)

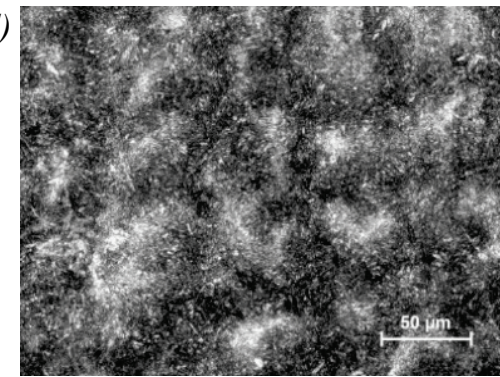

Fig. 3. Microstructure of C45 steel subjected to processing of: a) martensitic hardening, b) martensitic hardening and tempering at $250^{\circ} \mathrm{C}$, c) martensitic hardening and tempering at $400^{\circ} \mathrm{C}$

d) martensitic hardening and tempering at $600^{\circ} \mathrm{C}$

Figures 4 and 5 show examples of polarization plots for each of the samples. Tab. 2 contains the results of polarization measurements: corrosion potential $E_{k o r}[\mathrm{mV}]$ and corrosion current density $I_{k o r}\left[\mu \mathrm{A} / \mathrm{cm}^{2}\right]$.

In Fig. 6 is presented the relationship between heat treatment and $E_{k o r}$ potential. However, in Fig. 7 presents the relationship between heat treatment and the value of the $I_{k o r}$ corrosion current density. In Fig. 6, it can be seen that the highest value of corrosion potential was obtained after complete annealing and the lowest after martensitic hardening.

In Fig. 7, it can be seen that the highest value of the corrosion current density was obtained after martensitic hardening and the lowest after hardening and high tempering. The sample after martensitic hardening and low tempering deviates from the others. This is related to the phase structure of C45 steel, namely the cathodic precipitations of cementite. 


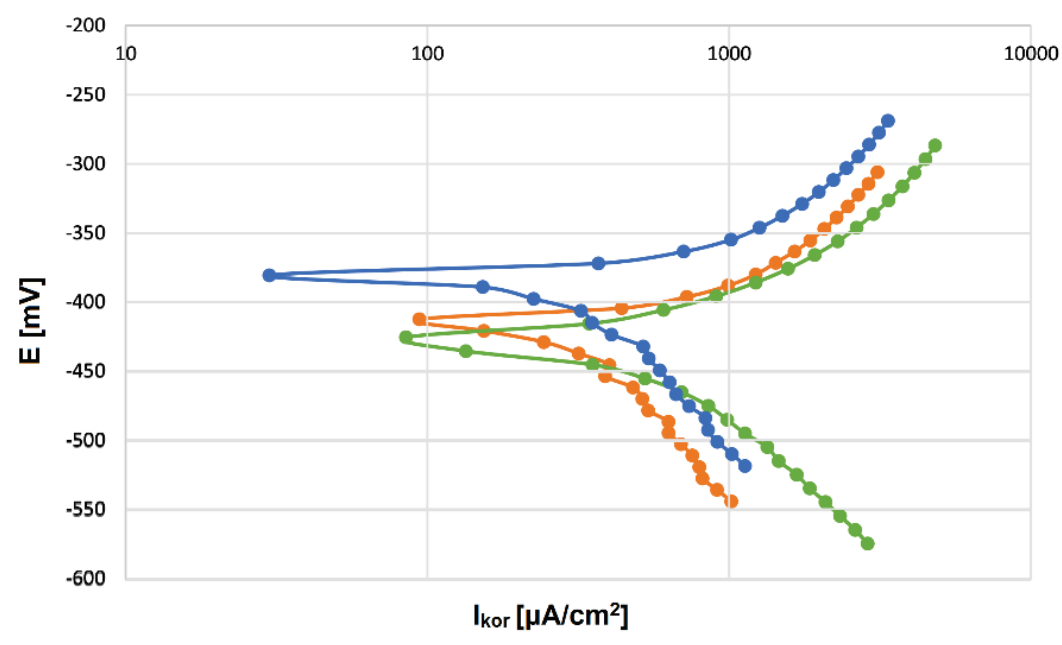

Fig. 4. Graph of polarization of samples after: orange line - incomplete annealing, green line-normalizing annealing, blue line-complete annealing

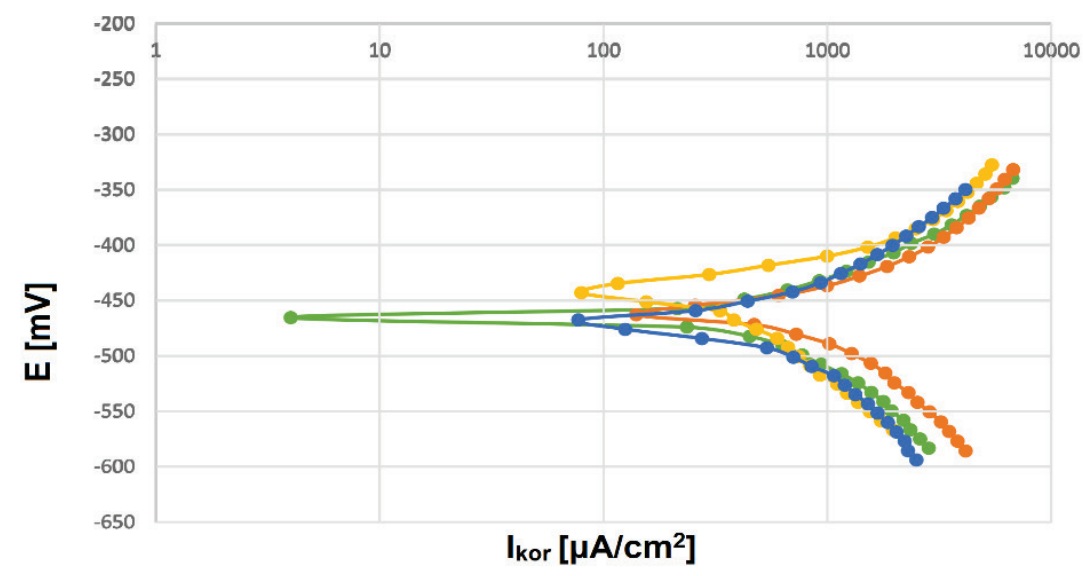

Fig. 5. Graph of polarization for the sample after: orange line-martensitic and low tempering, green line - martensitic hardening and medium tempering, yellow line-martensitic hardening and high tempering, blue line - martensitic hardening

Tab. 2. Determined values of: a) corrosion potential and b) corrosion current density

a)

\begin{tabular}{|c|c|c|c|c|c|c|c|}
\hline \multicolumn{8}{|c|}{$E_{k o r}[\mathrm{mV}]$} \\
\hline No. & W.N. & W.Z. & W.N.Z. & H.N.O. & H.Ś.O. & H.W.O. & H. \\
\hline 1. & -422 & -438 & -418 & -448 & -439 & -421 & -497 \\
\hline 2. & -422 & -362 & -419 & -460 & -488 & -426 & -460 \\
\hline Average value & -479 & -379 & -436 & -467 & -479 & -439 & -466 \\
\hline
\end{tabular}

b)

\begin{tabular}{|c|c|c|c|c|c|c|c|}
\hline \multicolumn{8}{|c|}{$\left.I_{\text {kor }}\left[\mu \mathrm{A} / \mathrm{cm}^{2}\right]\right]$} \\
\hline No. & W.N. & W.Z. & W.N.Z. & H.N.O. & H.Ś.O. & H.W.O. & H. \\
\hline 1. & 544 & 396 & 472 & 564 & 522 & 303 & 558 \\
\hline 2. & 506 & 420 & 440 & 556 & 580 & 283 & 596 \\
\hline 3. & 525 & 413 & 451 & 529 & 562 & 341 & 533 \\
\hline Average value & $\mathbf{5 2 5}$ & $\mathbf{4 1 0}$ & $\mathbf{4 5 4}$ & $\mathbf{5 5 0}$ & $\mathbf{5 5 5}$ & $\mathbf{3 0 9}$ & $\mathbf{5 6 2}$ \\
\hline
\end{tabular}

where: W.Z. - complete annealing, W.N.Z. - incomplete annealing, W.N. - normalizing annealing, H.W.O. - high tempered hardening, H.Ś.O. - medium tempered hardening, H.N.O. - low tempered hardening, H. - martensitic hardening. 


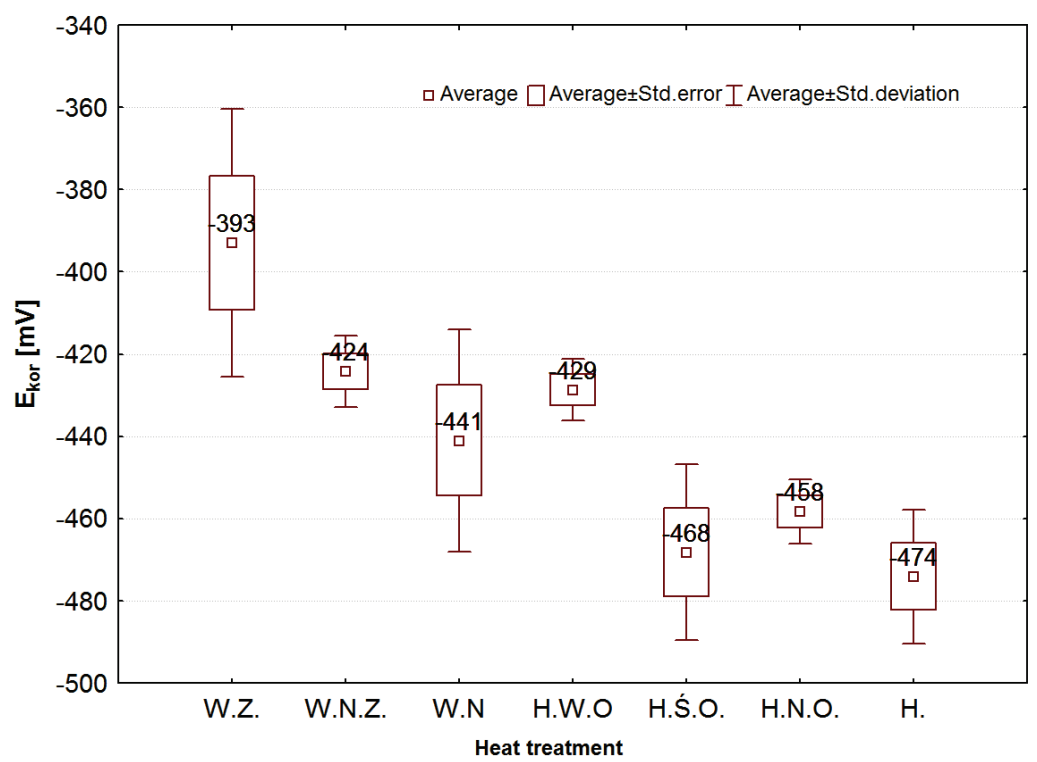

Fig. 6. Graph of the dependence between kind heat treatment used and corrosion potential

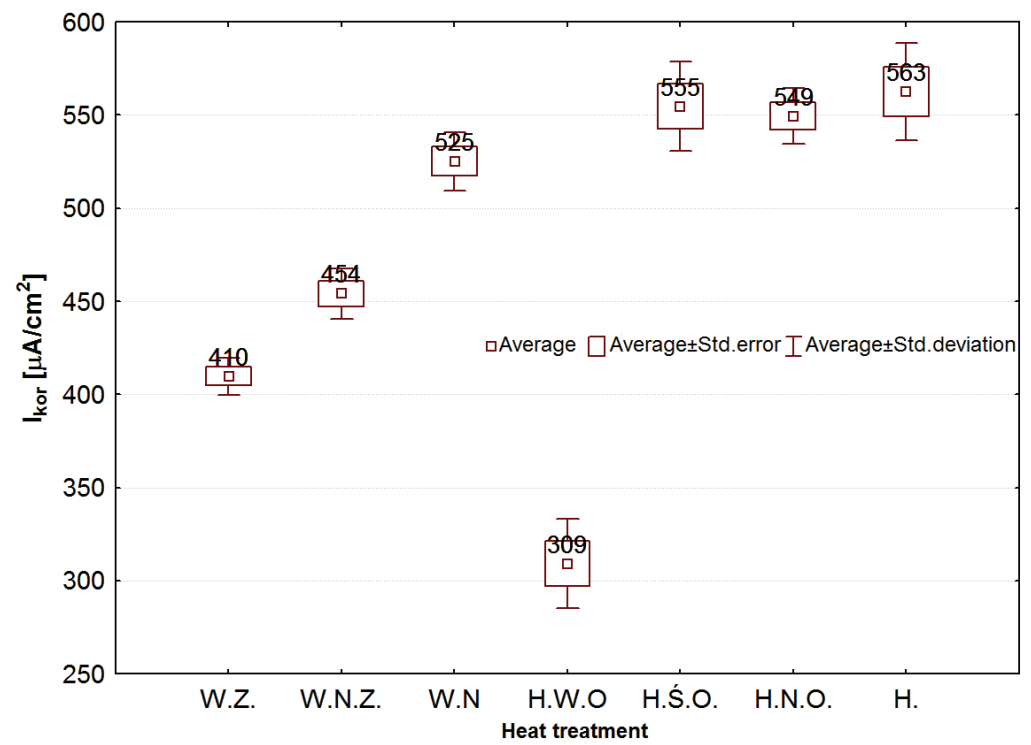

Fig. 7. Diagram of correlation between kind heat treatment used and corrosion current density

Figure 8 shows the dependence of hardness on the $E_{\text {kor }}$ potential. As the hardness increases the corrosion potential decreases.

A high negative correlation value $r=-0.84$ was found between the hardness of steel and its corrosion potential. On this basis, it can be concluded that the tested steel shows a greater tendency to environmental destruction as its hardness increases.

Figure 9 shows the dependence of hardness on corrosion current density. As the hardness increases the value of the corrosion, current density increases.

The value of the correlation coefficient is $r=0.66$. It can be concluded that the hardness of steel and the type of heat treatment operation affects the rate of corrosion of the steel being evaluated. From the results of the statistical analysis, it should be assumed that the rate of electrode oxidation reaction (anodic reaction) increases with the hardness of the steel. Analysing the graph in Fig. 9 can be notice large differences in the value of corrosion current density $\left(315-525 \mu \mathrm{A} / \mathrm{cm}^{2}\right)$ of samples with hardness in the range of $160 \mathrm{HV}$ and $230 \mathrm{HV}$. Which may indicate the influence of the microstructure (phase structure and grain size) on the corrosion rate. 


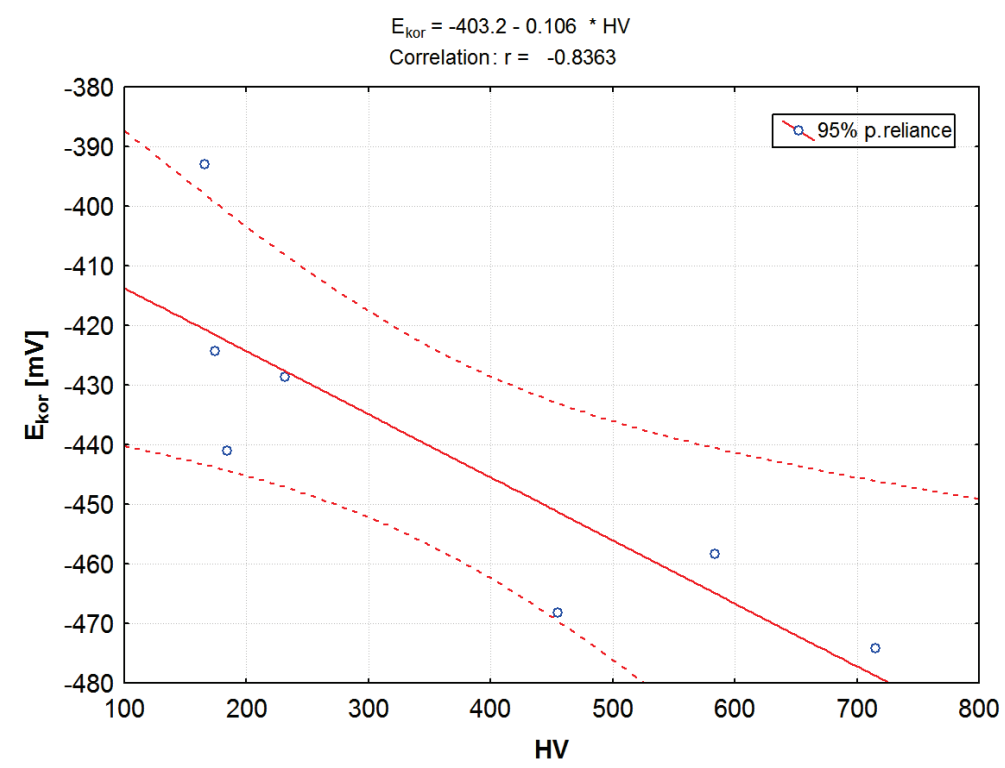

Fig. 8. Correlation between hardness and corrosion potential

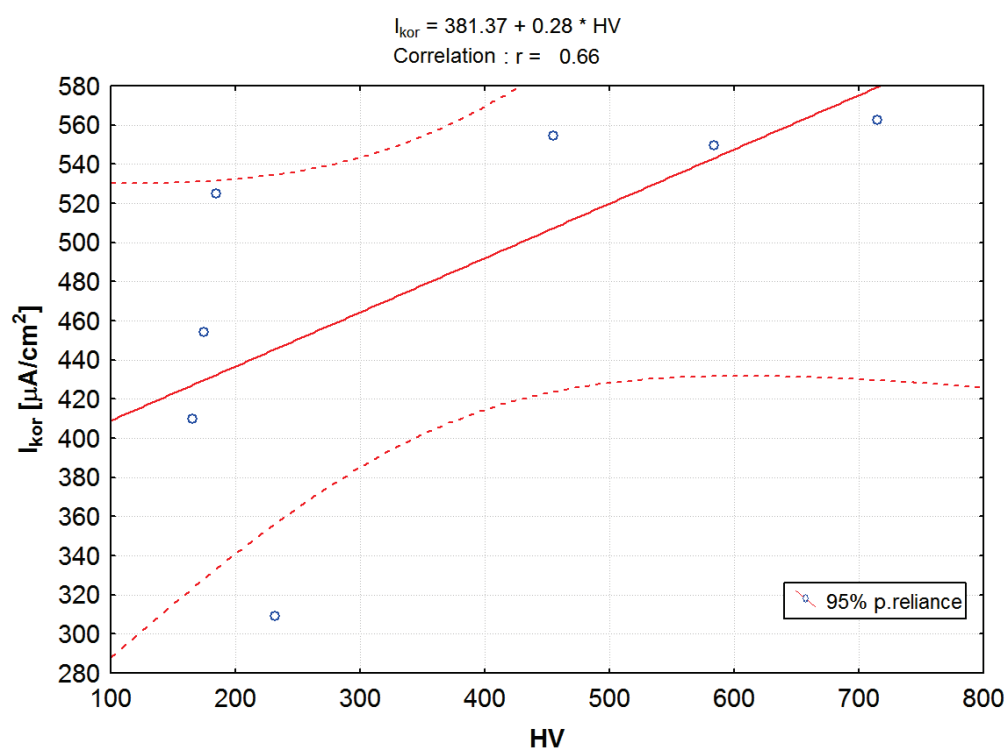

Fig. 9. Correlation between hardness and corrosion current density

\section{Summary and conclusions}

On the basis of the conducted tests, it can be concluded that the type of heat treatment carried out has an effect on the corrosion properties of non-alloyed $\mathrm{C} 45$ steel. There is a high negative correlation $(r=-0.84)$ between the hardness of the steel and the corrosion potential. Hardened material is characterized by a greater tendency to overlap on its surface of the oxidation reaction than the materials after annealing. The conducted linear regression analysis showed that the hardness of the material influences the speed of the electrode reactions $\left(I_{k o r}\right)$ on its surface. The harder the $\mathrm{C} 45$ steel, the faster the reactions take place.

\section{References}

[1] Bala, H., Corrosion of materials - theory and practice, Publishing House of the Faculty Process, Materials and Applied Physics Engineering, Czestochowa University of Technology, Czestochowa 2002. 
[2] Baszkiewicz, J., Kamiński, M., Principles of corrosion materials, Warsaw University of Technology, Warsaw 1997.

[3] Starosta, R., Fundamentals of manufacturing and processing of composite coating in the process of regeneration of machine elements and equipment operated in the sea water environment, Publisher of the Gdynia Maritime University, pp. 63, Gdynia 2013.

[4] Surowska, B., Selected problems of corrosion and corrosion protection, Publisher of the Lublin University of Technology, Lublin 2002.

[5] Trzaska, M., Trzaska, Z., Electrochemical impedance spectroscopy in material engineering, Warsaw University of Technology Publishing House, pp. 84-89, Warsaw 2010.

[6] Wesołowski, K., Metallurgy and heat treatment, WNT, Warsaw 1981. Manuscript received 17 June 2019; approved for printing 23 September 2019 